\title{
Experimental analysis of laser ablated plumes for asteroid deflection and exploitation
}

\author{
Alison Gibbings ${ }^{\mathrm{a}, \mathrm{b}, *}$, Massimiliano Vasile ${ }^{\mathrm{a}}$, Ian Watson ${ }^{\mathrm{b}}$, John-Mark Hopkins ${ }^{\mathrm{c}}$, David Burns ${ }^{\mathrm{c}}$ \\ a Advanced Space Concepts Laboratory, Department of Mechanical E' Aerospace Engineering, University of Strathclyde, 75 Montrose Street, Glasgow, UK \\ ${ }^{\mathrm{b}}$ Systems, Power and Energy Research Division, School of Engineering, University of Glasgow, James Watt (South) Building University Avenue, Glasgow, UK \\ ${ }^{\mathrm{c}}$ Institute of Photonics, University of Strathclyde, Wolfson Centre, 106 Rottenrow, Glasgow, UK
}

\section{A R T I C L E I N F O}

\section{Article history:}

Received 19 February 2012

Received in revised form

12 May 2012

Accepted 7 July 2012

Keywords:

Laser ablation

Asteroid exploitation

Asteroid deflection

\begin{abstract}
A B S T R A C T
It has been theoretically demonstrated that laser ablation is effective in the potential deflection and mitigation of asteroids. However, there have been few experimental studies to support this claim. The theoretical models are currently based on assumptions regarding the laser beam diameter, the power requirement, the formation of the ejecta plume, and the potential for ejecta to contaminate and otherwise degrade any exposed surface. Recent proposals suggesting the use of a solar pumped laser, in particular, can be deeply affected by the re-condensation of the ejecta. To either validate, amend and/or eliminate these assumptions a series of laser ablation experiments have been performed. Using a $90 \mathrm{~W}$, continuous-wave laser operating at $808 \mathrm{~nm}$, a rocky magnesium iron silica based material - olivine - has been ablated. These experiments were used to examine the validity of the theoretical model and the experienced levels of contamination. It will be shown that the current model correctly predicts the ablated mass flow rate for rocky based asteroids, but overestimates the contamination rate and the degradation of the optics.
\end{abstract}

(c) 2012 Elsevier Ltd. All rights reserved.

\section{Introduction}

Near Earth Asteroids (NEAs) represent both an opportunity and a risk. Their pristine environment captures the early formation of the solar system; while their impact potential could result in the mass extinction of life. The Earth has been, and will continue to be, the subject of many other ground and air impacting events. Amid the observed population, there are at least between 2000 and 200,000 objects that could impact the Earth [1]. On average, an asteroid with a diameter greater than $100 \mathrm{~m}$ impacts the

\footnotetext{
* Corresponding author at: University of Strathclyde Department of Mechanical \& Aerospace Engineering Advanced Space Concepts Laboratory 75 Montrose Street, Glasgow G1 1XJ, United Kingdom.

Tel.: +4407795060527.

E-mail addresses: a.gibbings.1@research.gla.ac.uk, alison.gibbings@strath.ac.uk (A. Gibbings), massimiliano.vasile@strath.ac.uk (M. Vasile),

i.watson@glasgow.ac.uk (I. Watson), johnmark.hopkins@strath.ac.uk (J.-M. Hopkins), d.burns@strath.ac.uk (D. Burns).
}

Earth once every 10,000 years. This can cause local damage, earthquakes and tsunamis. Asteroids that impact the Earth with a diameter larger than $1 \mathrm{~km}$ are considered to be global killers. Such an impact event is considered to catastrophically annihilate $90 \%$ of all life, resulting in a nuclear winter, with little chance of recovery within the near term [1]. This is thought to have happened, once before, approximately 65 million years ago, with the impact of a $10 \mathrm{~km}$ diameter asteroid at $12 \mathrm{~km} / \mathrm{s}$ [23].

Therefore potential methods of asteroid mitigation and deflection have been addressed by numerous authors [2-4]. Amongst the many possibilities to deflect NEAs, ablation has been shown to be theoretically one of the most effective methods [5]. Work conducted in 2009 by Sanchez et al. [5] compared the effectiveness of six different asteroid deflection techniques. Through a multi-criteria, quantitative comparison the nuclear interceptor, kinetic impactor, mass driver, low thrust tug, ablation and the gravity tractor were assessed. Assessment was made relative to the achievable miss distance at 


\section{Nomenclature}

$(\Delta m / A)_{\text {SLIDES }}$ Ejecta mass per unit area deposited on the microscope slides during ablation

A Area of the microscope slide

$a_{b} \quad$ Absorbance

$A_{S P O T} \quad$ Area of the laser's surface spot

$c_{A} \quad$ Heat capacity of the asteroid

$C_{m} \quad$ Momentum coupling

$C_{R} \quad$ Concentration ratio

$d_{S P O T} \quad$ Diameter of the surface spot

$d h / d t \quad$ Surface layer growth

$d m / d t \quad$ Mass flow rate during sublimation

$E \quad$ Incoming energy during the ablation process

$E_{v} \quad$ Sublimation enthalpy of the asteroid

$F_{S U B} \quad$ Force acting on the asteroid

$h_{E X P} \quad$ Height of the deposited ejecta from the experiment

$k \quad$ Adiabatic index, for diatomic molecules

$k_{b} \quad$ Boltzman's constant

$K_{p} \quad$ Jet constant

$k_{t} \quad$ Thermal conductivity of the asteroid

$M_{a} \quad$ Molar mass of the target material

NEAs Near Earth Asteroids

$P_{1 \mathrm{AU}} \quad$ Solar power at 1 Astronomical Unit

$P_{I N} \quad$ Absorption of the laser beam

$Q^{*} \quad$ Energy required to ablated each kilogram of material

$Q_{\text {COND }} \quad$ Heat losses due to conduction

$Q_{R A D} \quad$ Heat losses due to radiation
$R$

$r$

SEM Scanning Electron Microscope

$t \quad$ Sublimation duration

$T \quad$ Transmittance

$T_{a m b} \quad$ Ambient temperature

$T_{0} \quad$ Temperature at the centre of the asteroid

$T_{S U B} \quad$ Sublimation temperature of the asteroid in vacuum conditions

$v \quad$ Average velocity of the ejecta plume

$\varepsilon \quad$ Black body emissivity of the asteroid

$\eta \quad$ Absorptivity

$\eta_{A B} \quad$ Efficiency of the ablation process

$\theta \quad$ Elevation angle, from the surface normal

$\theta_{\text {MAX }} \quad$ Limited expansion angle

$\lambda \quad$ Scatter factor

$\rho \quad$ Density of the ejecta plume

$\rho^{*} \quad$ Density at the nozzle

$\rho_{A} \quad$ Density of the asteroid

$\rho_{1} \quad$ Layer density

$\sigma_{S B} \quad$ Stefan-Boltzmann constant

$\tau \quad$ Degradation factor

$\psi_{v f} \quad$ View angle

$\Delta h_{E X P} \quad$ Measured thickness of the deposited material

$\Delta m \quad$ Mass loss during ablation

$\Delta t \quad$ Ablation duration

$\eta_{\text {sys }} \quad$ Overall conversion efficiency from solar input to laser output

$\eta_{a b s} \quad$ Absorptivity of the illuminated asteroid
Earth, the warning time, the total mass into orbit and the current technology readiness level. With both a relatively short warning time and a low mass into space, ablation can provide significantly higher and more controllable rates of deflection. The technique is also advantageous as it avoids the catastrophic fragmentation of the asteroid. It also eliminates the need of having to physically land and/ or attach a system onto the surface of the asteroid [5].

Ablation is achieved by irradiating the asteroid with a light source. This can either be collected and focussed solar radiation or with a laser light source. Within the illuminated focal point, the absorbed energy increases the temperature of the asteroid, enabling it to sublimate. This transforms the exposed material directly from a solid to a gas. The ablated material then expands to form an ejecta plume. Over an extended period of time, the resultant thrust, induced by the ejecta plume and acting on the asteroid can be used to push the asteroid away from its original threatening trajectory [2]. This increases the minimum orbit interception distance between the Earth and the asteroid, otherwise preventing the Earth impacting event [5-8].

Previous proposals for the initiation of laser ablation considered using either a ground-based or space-based facility $[1,19]$. For a ground-based facility an average power level of several giga-watts would be required to deflect a small, 40-80 $\mathrm{m}$ in diameter asteroid [19]. This was considered to be a substantial investment in infrastructure and resources. Therefore an alternative option was to mount a mega-watt laser onto a large single spacecraft. The laser would be powered by a nuclear reactor [20]. However, manoeuvring and operating such a large structure, at close proximity to the asteroid, under an irregular gravity field was considered to be very difficult. This is further coupled with developing a nuclear reactor for space-based applications, and the associated political ramifications. Therefore an alternative concept was proposed. Instead of a large single structure, a swarm of small spacecraft, each equipped with an identical kilo-watt solar-pumped laser could be used [10]. This provides a much lighter and more adaptable concept. By superimposing each laser beam, the cumulative surface power density would be used to initiate the ablation process [10]. Singular or multiple ablation spots can also be used. This increases the flexibility and overall redundancy of the deflection mission. As required, more spacecraft can be added or removed from the existing configuration, eliminating the need to develop and design new spacecraft(s) $[6,7,9]$. The potential for deflection is therefore dependent on the number of spacecraft located within the vicinity of the asteroid, their combined laser power and the material properties of the asteroid.

Within this configuration, each laser would be powered by the Sun, either directly or indirectly. For direct pumping the solar radiation is collected and concentrated directly onto the laser medium. For indirect pumping, the incoming solar 
radiation is first focussed onto a set of highly efficient solar cells. This immediate step is used to convert the incoming solar radiation into electrical energy that is then used to power the laser. For both cases - direct and indirect pumping - a light, deployable primary mirror and a smaller secondary (both known as the solar concentrators) are needed to collect the freely available solar radiation of the Sun. A steering mirror is also required to target the laser beam onto the surface of the asteroid. Large radiators are needed to keep the laser within its operational limit and to cool the spacecraft.

However, any exposed surface within the vicinity of the ejecta volume, including the steering mirror and solar concentrators, would be subjected to the continual contaminating effects of the condensing ejecta. It is currently assumed that once the ejecta plume comes into contact with any given surface that the particles will immediately re-condense and stick. The continued accumulation of the ejecta will decrease the transmittance and increase the absorbance of the exposed surface. The degradation caused by the ejecta is considered to follow the BeerLambert-Bouguer law and is dependent on the absorptivity of the condensed material. The laser beam is also expected to be attenuated by the ablated plume of ejecta.

This paper will show that the effect of the ejecta contamination, according to the current model, has a major impact on the ability of the laser ablation process to produce a significant deflection action. Therefore to examine the actual operational and environmental constraints of laser ablation, a series of experiments have been performed. Within a vacuum chamber, and using a $90 \mathrm{~W}$ continuouswave laser, the ablation response of a magnesium iron silicate (olivine) sample has been assessed. This assessment included the development of the ejecta - cone angle divergence, mass flow rate, and plume density - and the affects of the condensing ejecta. All results have been compared to the numerical model. This paper therefore investigates the effect of laser irradiation on a rocky based asteroid simulate. Providing experimental data and model validation is an important step towards the realisation of a laser asteroid deflection system. This paper details the current ablation and asteroid deflection models; highlighting the conditions placed upon the assumed physical parameters and the response of the ablation process. The laser ablation experiment is then presented. The results from the experiment are then given and placed within the context of a revised deflection simulation. Conclusions have been drawn and areas of future work addressed.

\section{Current ablation and asteroid deflection models}

The current ablation model is based on the energy balance of sublimation [6,11]. This combines the absorbed laser beam power $P_{I N}$, the sublimation enthalpy of the illumined material by the laser $E_{v}$, and the heat losses through conduction $Q_{C O N D}$ and radiation $Q_{R A D}$ respectively. Therefore the mass flow rate $\dot{m}_{\exp }$ of the ablated material during sublimation can be expressed as

$\frac{d m}{d t}=\dot{m}_{\exp }=\frac{1}{E_{v}}\left(P_{I N}-Q_{R A D}-Q_{C O N D}\right)$
If the mass flow is negative then there is not enough energy to initiate the ablation process.

The heat loss through radiation was assumed to act as a black-body and is therefore defined as

$Q_{R A D}=\sigma_{S B} \varepsilon A_{S P O T}\left(T_{S U B}^{4}-T_{a m b}^{4}\right)$

where $\sigma_{S B}$ is the Stefan-Boltzmann constant (5.6704 $\left.10^{-8} \mathrm{~W} / \mathrm{m}^{2} \mathrm{~K}^{4}\right), \varepsilon$ is the black body emissivity of the illuminated material, $A_{S P O T}$ is the area of the spot illuminated by the laser, $T_{S U B}$ is the sublimation temperature of the illuminated material (in vacuum conditions) and $T_{a m b}$ is the ambient temperature that in space is $4 \mathrm{~K}$ and in the laboratory environment is $298 \mathrm{~K}$.

The heat loss due to conduction is determined from

$Q_{\text {COND }}=\left(T_{S U B}-T_{0}\right) A_{S P O T} \sqrt{\frac{c_{A} \rho_{A} \kappa_{t}}{\pi t}}$

where $c_{A}, \rho_{A}$ and $k_{t}$ are the heat capacity, density and thermal conductivity of the asteroid respectively, $t$ is the equivalent time to bring a layer ( $<1 \mathrm{~mm}$ thick) of the target material to sublimation temperature (this depends on the diffusion in the target material and the speed of recession of the ablatedejecta layer), and $T_{0}$ is the temperature at the centre of the asteroid. This is assumed to be $298 \mathrm{~K}$, which coincides with the local temperature of the laboratory. For the simulations within this paper, the density of the asteroid analogue target material was measured. All other values were assumed based on the physical properties of olivine. This is given in Table 1 . The enthalpy of sublimation, as given in Table 1, combines the enthalpy of vaporisation and the heat fusion. It is considered to be the complete enthalpy of vaporisation, rather than the incident of vaporisation.

Note that, the model assumes that the internal temperature of the asteroid is unchanged and therefore remains at $298 \mathrm{~K}$ throughout the ablation process. Furthermore, the asteroid is not rotating. The internal temperature corresponds to the constant and controlled room temperature of the laboratory. The latter assumption is introduced for consistency with the experiments that will be conducted on a non-rotating target.

The average velocity of the ejecta plume $\bar{v}$ can be calculated assuming Maxwell's distribution of an ideal gas $[5,6]$. This is defined by the sublimation temperature $T_{S U B}$, the molar mass of the ablated gaseous phase of the target material (this is taken to be $50 \mathrm{~g} / \mathrm{mole}$ and accounts for a diatomic molecule of forsterite within the vapour regime) $M_{a}$, and Boltzman's constant $k_{b}$. This is given by

$\bar{v}=\sqrt{\frac{8 k_{b} T_{S U B}}{\pi M_{a}}}$

Table 1

Assumed parameters for the asteroid analogue target material.

\begin{tabular}{ll}
\hline Parameter & Value \\
\hline Density $\left(\mathrm{kg} / \mathrm{m}^{3}\right)$ & 3500 \\
Sublimation enthalpy, $E_{v}(\mathrm{~J} / \mathrm{kg})[26]$ & $14.5 * 10$ \\
Black body emissivity, $\varepsilon[6]$ & 0.95 \\
Temperature at the centre of the asteroid, $T_{0}(\mathrm{~K})$ & 298 \\
Heat capacity, $c_{A}(\mathrm{~J} / \mathrm{kgK})$ & 1361 \\
Thermal conductivity, $k_{\mathrm{A}}(\mathrm{W} / \mathrm{mK})[25]$ & 4.76
\end{tabular}


The force acting on the asteroid $F_{\mathrm{SUB}}$ is given by the product of the ejecta velocity and the mass flow rate of the ablated material. This is given as

$F_{S U B}=\lambda \bar{v} \dot{m}_{\exp }$

A constant scatter factor $\lambda$ is used to account for the uniform expansion of the ejecta over a given half sphere. This is considered to be a worst case, conservative assumption. The velocity of the ejecta is also assumed to be constant, independent of the local elevation angle, and therefore is only a function of the sublimation temperature. This is given in Eq. (4). The force acting on the asteroid is consequently only dependent on the mass flow rate. A higher mass flow rate results in a higher thrust.

The input power $P_{I N}$ is computed assuming that the beam is generated by pumping a fibre laser. The electric power is generated by a solar concentrator focussing the sunlight onto a solar array. It is assumed that only $80 \%$ of the incoming laser light is absorbed and that the rest is reflected. The absorbed power is therefore

$P_{I N}=\tau \eta_{a b s} \eta_{s y s} C_{R} \frac{P_{1 A U} A_{S P O T}}{R^{2}}$

where $\tau$ is the degradation factor due to contamination (see below), $\eta_{\text {abs }}$ is the absorptivity of the illuminated asteroid, $\eta_{\text {sys }}$ is the overall conversion efficiency from the solar input to the laser output, $C_{R}$ is the concentration ratio or ratio between the area of the solar concentrator and the area of the spot, $P_{1 \mathrm{AU}}=1378 \mathrm{~W} / \mathrm{m}^{2}$ is the solar power at 1 Astronomical Unit, $A_{\mathrm{SPOT}}$ is the area of spot on the surface of the asteroid, and $R$ is the distance from the Sun measured in Astronomical Units. The overall conversion efficiency is assumed to be $19 \%$. This accounts for solar cells with an efficiency of $40 \%$, a laser system with an efficiency of $60 \%$ and a nominal reflectivity of the concentrators of $90 \%$.

The sublimation process is also considered to be governed by three fundamental assumptions. The first considers that the formation of the ejecta plume is similar to, although not identical to, the development of a rocket exhaust in standard methods of rocket propulsion. The same approach is used to model cometary sublimation [12]. This assumes that the ablated ejecta is a compressible, friction free gas (with no solid particles), which has a constant velocity and temperature, and occurs below the limit of ionisation of plasma formation [11,6]. It therefore assumes a vapour-only flow regime. At increased intensities a strong laser generated plasma can form. Plasma, through the inverse Bremsstrahlung effect, is known to absorb the incoming laser beam and therefore shield the target from its affects. This would dramatically reduce the coupling between the laser beam and the asteroid that otherwise provides the low thrust required for the deflection technique to become effective. The second assumption is that the asteroid is a spherical, dense, non-porous homogenous body. The asteroid is considered to have an infinite heat sink, where during the ablation process the asteroid maintains a constant internal temperature. The third assumption is that all the ejected particles will immediately re-condense and stick to any exposure surface. Degradation is assumed to follow

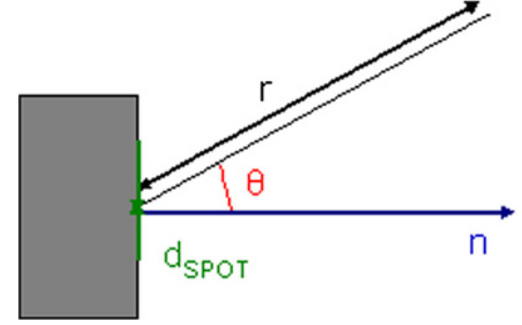

Fig. 1. Geometry of the plume-local reference frame.

the Beer-Lambert-Bouguer law, and a sticky coefficient of one is used throughout $[11,6]$.

By taking the first assumption the density of the ejecta plume at any given distance $r$ from the spot location, and elevation angle $\theta$ from the surface normal can be defined [11]. This is illustrated in Fig. 1 and can be expressed as

$\rho(r, \theta)=\rho^{*} K_{P} \frac{d_{S P O T}^{2}}{\left(2 r+d_{S P O T}\right)^{2}}\left[\cos \left(\frac{\pi \theta}{2 \theta_{\mathrm{MAX}}}\right)\right]^{2 / k-1}$

A compressible, friction free gas is typically classified with a constant adiabatic index $k$, where for diatomic molecules $k=1.44$ [11]. In Eq. (7) the jet constant $K_{p}$ for diatomic molecules is defined as 0.345 with the limited expansion angle $\theta_{\text {MAX }}$ as $130.45^{\circ}$ [24]. By considering the standard rocket equation, the density at the nozzle, $\rho^{*}$ is also given as

$\rho^{*}=\frac{\dot{m}_{\exp }}{A_{S P O T} \bar{v}}$

Kahle et al., 2006 [11] also considered the rate of ejecta contaminating and degrading any exposed surface. The variation of ejecta thickness - known as the surface layer growth - on any exposed surface can be expressed as $[6,11]$

$\frac{d h}{d t}=\frac{2 \bar{v} \rho}{\rho_{l}} \cos \psi_{v f}$

where $\psi_{v f}$ is the view angle i.e. the angle between the normal to the surface and the surface-to-spot vector. To account for the expansion of gas into a vacuum the average velocity is multiplied by a factor of two. The denominator $\rho_{l}$ is the layer density. Based on current literature, for a magnesium iron silica material, this is assumed to be $1 \mathrm{~g} / \mathrm{cm}^{3}$ [11]. All of the ablated particles of ejecta coming from the asteroid are assumed to stick to every exposed surface on the spacecraft. The increasing thickness of the contaminants will ultimately reduce the reflectivity of the concentrators and therefore the laser output power. The consequence is a reduction of the thrust imparted onto the asteroid until the sublimation ceases completely and the thrust associated with it. The reduction of the reflectivity of the concentrator, known as the degradation factor, $\tau$ can be computed from the BeerLambert-Bouguer law [11]. This is given by

$\tau=e^{-2 \eta h}$

where $\eta$ is the absorptivity (absorbance per unit length) of the accumulated ejecta. For olivine, at $808 \mathrm{~nm}$ (the wavelength of the laser) this is assumed to be approximately $10^{4} /$ $\mathrm{cm}[11,10]$. A factor of 2 within the expression accounts for the double passing of the surface layer [11]. 
Gauss planetary equations are used to compute how the sublimation process will affect the orbit of the asteroid. It is assumed that the force in Eq. (5) is always aligned to the velocity vector of the asteroid [18]. The Gauss planetary equations are propagated forward in time from the moment the sublimation starts to the expected time of the impact of the asteroid with the Earth [18]. At the expected time of impact with the Earth, this can be used to compute the position and velocity of the deflected asteroid. From the position and velocity, the impact parameter at the expected time of impact can be computed.

For example, for a relatively small NEA, with an assumed diameter of $250 \mathrm{~m}$ and a mass of $2.7 \cdot 10^{10} \mathrm{~kg}$, the miss distance is illustrated in Fig. 2. For this case, multiple spacecraft are flying in formation with the asteroid. Each spacecraft carries a laser (with an output power of $22 \mathrm{~kW}$ ) and a $10 \mathrm{~m}$ diameter primary mirror (solar concentrator). Together they concurrently beam their lasers onto the same spot on the surface of the asteroid. It is assumed that the temperature of the spot remains constant and is equal to the assumed sublimation temperature of Forsterite, which is $1800 \mathrm{~K}$. Fig. 2 shows the miss distance as a function of the number of spacecraft and the warning time. The miss distance, as given in Fig 2, is shown in kilometers. The warning time is the time from the beginning of the deflection action to the expected impact of the asteroid with the Earth. The result in Fig. 2 does not include the effects of contamination. With deflection operations of between 1 and 9 years (from the expected impact with the Earth) Fig. 2 demonstrates an achievable miss distance of between 5000 and $30,000 \mathrm{~km}$ respectively.

Fig. 3 shows the result for the same mission scenario but this time including the contamination of the solar concentrator. This is in accordance with the model developed by Kahle et al., 2006 [11]. The contamination induces a fast degradation of the collected power and a rapid halt of the sublimation process. Depending on when the sublimation starts and ends the miss distance can increase or decrease (see Colombo et al., 2008 for more

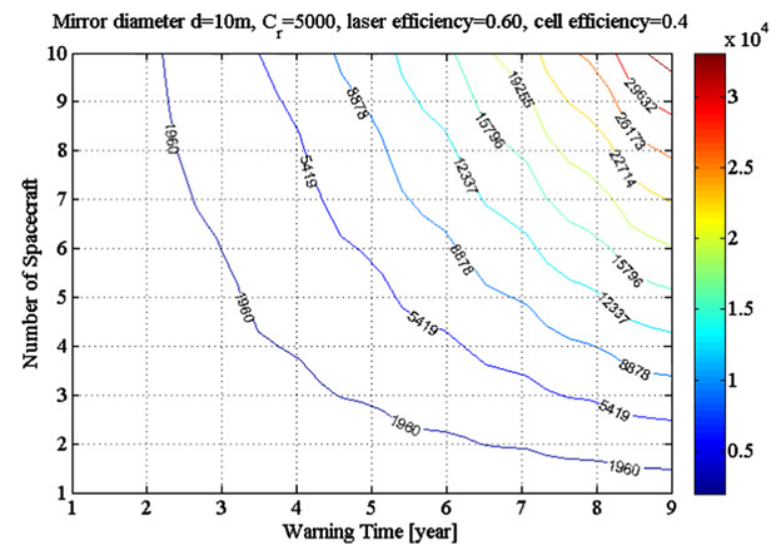

Fig. 2. Miss distance of a $250 \mathrm{~m}$ diameter, $2.7 \cdot 10^{10} \mathrm{~kg}$ (based on Apophis) asteroid as a function of the warning time and the number of satellites: without contamination.

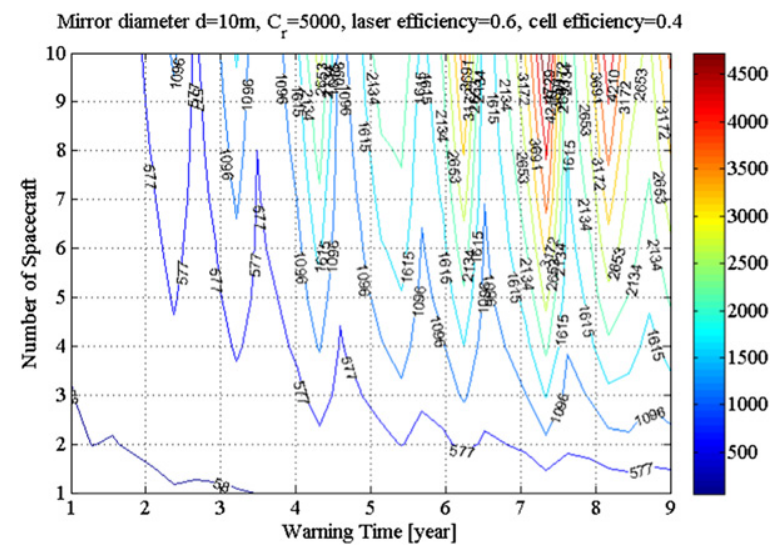

Fig. 3. Miss distance of a $250 \mathrm{~m}$ diameter, $2.7 \cdot 10^{10} \mathrm{~kg}$ (based on Apophis) asteroid as a function of the warning time and the number of satellites: with contamination.

details [18]). This is the reason for the periodic trend with the warning time that can be seen in Fig. 3 .

As illustrated in Fig. 3, accounting for ejecta degradation and contamination, the maximum achievable miss distance reduces to $4500 \mathrm{~m}$. This results in a significant reduction of performance of $85 \%$. Contamination reduces the achievable surface power density of the laser, and the associated thrust imparted onto the asteroid. Contamination of any optical surface will have a major impact on the overall success of any asteroid deflection mission.

The model developed in this section also allows for the estimation of the coupling between the output power from the laser and the change of linear momentum of the asteroid. This is known as the momentum coupling coefficient, $C_{m}$ [13-16]. For continuous laser operations it is defined as the induced force $F_{S U B}$ relative to the incoming absorbed laser power that occurs at the spot location. This can be written as:

$C_{m}=\frac{F_{S U B}}{P_{I N_{E X P}}}$

During the experiment the force was not directly measured, but was calculated from Eq. (5) combining the measured ablated mass flow rate with the measured ejecta gas velocity. The absorbed power $P_{I N_{E X P}}$ was taken from the known power illumination of the spot and combined with the assumed absorption of the target material and the laser beam within the plume. The energy required to ablate each kilogram of asteroid material $Q^{*}$ could also be determined. This is measured in $\mathrm{J} / \mathrm{kg}$ and is expressed as

$Q^{*}=\frac{E}{\Delta m}$

The energy $E$ is the incoming laser energy during the ablation process. It is therefore a product of the incoming absorbed laser power at the spot location multiplied by the ablation time. $\Delta m$ is the mass of the ablated material in a given time period. From this and the momentum coupling, the efficiency of the ablation process $\eta_{A B}$ could be determined. This defines the efficiency at which the laser energy is converted into kinetic energy, carried away 
by the ablating ejecta. This is given as $[14,21]$

$\eta_{A B}=\frac{1}{2} C_{m}^{2} Q^{*}$.

Note that $\eta_{A B}$ does not include the effect of any absorption losses within the ejecta plume. It is based purely on the incoming laser energy and power at the spot location.

\section{The experiment}

A $90 \mathrm{~W}$ continuous-wave laser, operating at $808 \mathrm{~nm}$, was used to initiate all ablation events. This was below the threshold of plasma formation. The formation of plasma was not accounted for within the model, and would otherwise have a degrading effect on the quality and efficiency of the ablation process $[14,15,6]$. For initial calibration, all ablation events occurred within a sealed and self contained glass test chamber. The chamber was purged with nitrogen to reduce the occurrence of atmospheric combustion to negligible levels. The ejecta mass flow rate, plume density, plume divergence and the influence of the re-condensing ejecta were then assessed [22]. The experiment was then repeated within a vacuum chamber. A pump down pressure of $2 \cdot 10^{-5}$ mbar was achieved. This removed the atmospheric particle drag disturbance; allowing for the maximum and free spreading of the plume [17]. This was considered to provide a realistic simulation of the laser-to-asteroid interaction.

Olivine was used to represent a dense, rocky, s-type asteroid. It had a density of $3500 \mathrm{~kg} / \mathrm{m}^{3}$ and was shaped into a cube by cutting a larger rock sample with a diamond blade. This enabled all ablation events to occur onto a flat face. A flat face was used to avoid, or severely limit, the ablation process being subjected to any irregularity of the surface material (including surface curvature) and any inhomogeneities of the target material's composition. The aim was to provide a tightly focussed laser beam onto the surface of the target material. It was also considered to be a realistic analogue of the in-space event, where the spot size of the laser beam would be small in comparison to the size, and major features on the surface of the asteroid. Mass measurements of the target material before and after each ablation event enabled the rate of sublimation to be determined.

The test chamber was surrounded by two cameras, and a spectrometer. Viewing access was granted through optical windows. Each window had a transmittance of approximately $94 \%$. Two high resolution, digital cameras (Panasonic HDC), mounted perpendicular to each other, recorded each ablation event. During each ablation event the cameras were used to measure the divergence and formation of the ejecta plume. A spectrometer was used to measure the inferred temperature of the ablated gas. This was determined from the Wien's Displacement law, and was achieved by measuring the intensity and wavelength of the emitted spectra. The velocity of the gaseous ejecta was then calculated, assuming Maxwell's distribution of an ideal gas. All experiments were repeated three times. This aimed to provide more viable and well calibrated data points.
Within the chamber, the asteroid analogue target material was mounted on a raised pedestal, at a predetermined location. This was relative to the known focal point of the laser, which had an approximate spot size diameter of $\sim 1.8 \mathrm{~mm}$. This corresponded with a surface power density (accounting for optical losses through the system) of $\sim 2.44-1.69 \mathrm{~kW} / \mathrm{cm}^{2}$. For each experiment reported herein, ablation occurred for $10 \mathrm{~min}$. To collect and assess the ablated ejecta, highly cleaned microscope slides were positioned within the ablation volume. Before and after each ablation event, mass measurements of the microscope slides enabled the mass of the deposited ejecta at different points within the plume to be determined. By measuring the mass per unit area deposited on each microscope slide $(\Delta m / A)_{\text {SLIDES, }}$ where $A$ is the area of the microscope slide, and by measuring the thickness of the deposited material $\Delta h_{\mathrm{EXP}}$, the density of the deposited material can be computed. This is achieved by

$\rho_{\text {EXP }}(r, \theta)=\frac{\left(\frac{\Delta m(r, \theta)}{A}\right)_{\text {SLIDES }}}{\Delta h_{\text {EXP }}}$

From the model, the expected collection rate of the ejecta on each microscope slide can also be derived. This is given as

$\frac{1}{A} \frac{d m}{d t}=2 \rho(r, \theta) \bar{v}$

Eq. (15) assumes that the velocity of the expanded gas is about $2 \bar{v}$ (to account for full expansion into a vacuum) and that all the particles are sticking onto the surface of the microscope slides. The effect of the deposited ejecta mass was assessed by measuring the light transmittance across each microscope slide. The relative reduction in the transmittance $T$ and the increase in absorbance $a_{b}$ caused by the deposited ejecta were measured. This was achieved by either using a power meter or photographic analysis of the actual microscope slides. By considering only a one way transmittance of the microscope slides, the value of the associated absorbance can be calculated from the Beer-Lambert-Bouguer law for optical absorption. This is given as

$a_{b}=\log \frac{1}{T}$

Given the absorbance $a_{b}$, the associated absorptivity $\eta_{\text {EXP }}$ (absorbance per unit length) given in Eq. (10) can be determined. This is achieved by dividing the experimentally determined absorbance $a_{b}$ by the experimentally measured height of the deposited ejecta $h_{\text {ExP. The height }}$ of the collected ejecta was measured with a Nikon Nomarski microscope. Therefore

$\eta_{\text {EXP }}=\frac{a_{b}}{h_{E X P}}$

In the following section the absorptivity of the contamination layer estimated from the experiments will be used to re-assess the effectiveness of the deflection action. The degradation is a function of both the absorptivity of the condensed layer and the thickness, which in turns depends on the layer growth rate. By measuring $h_{E X P}$ over time one can also derive a correction factor to Eq. (10) and 
compute a degradation factor that is consistent with the experimental results.

\section{Results}

Laser ablation resulted in the formation of a small and extended rocket plume. This was dominated by the gaseous exhaust of material, and is illustrated in Fig. 4. From the initial illumination of the laser beam, in addition to the gaseous exhaust, the ablation process also resulted in the formation of small solid particles. However, throughout all the experiments this only occurred for a very small portion of time; almost instantaneously once the laser beam had initially illuminated the olivine sample. The solid particles were also distributed over $180^{\circ}$. This is also illustrated in Fig. 4, and is portrayed by the creation of white streaks. The generation of solid ejecta is not currently accounted for within the numerical model, and would otherwise enhance the momentum exchange between the laser beam and target material.

Under high vacuum, based on the model with an input illumination power of either 62 or $43 \mathrm{~W}$, the initial mass flow of the ablated ejecta was $2.10^{-7} \mathrm{~kg} / \mathrm{s}$. At the end of the ablation period (10 $\mathrm{min}$ ) the experimental mass flow rate of the ablated material had reduced to $2.5 \times 10^{-8} \mathrm{~kg} / \mathrm{s}$. The rapid reduction in the mass flow rate is expected to be due, in part, due to the defocusing of the laser beam and the additional thermal effects that are not currently accounted for within the numerical model. Critically the model does not account for the three dimensional thermal diffusivity. It is limited to a mono-dimensional transfer of heat. It also assumed a constant value of emissivity, heat capacity, density and thermal conductivity. In reality these parameters have a temperature dependence on the optical and thermal properties of the target material [29].

In practice the ablation response resulted in the widening and deepening of the surface hole. This was caused by the tunnelling away of the surface and subsurface material. A small, narrow hole was formed that extended into the target material. Similar to a rocket exhaust, as a function of time, this would have assisted in constraining the formation of the gaseous ejecta plume. The surface hole diameter was also widened slightly. An initial surface spot radius of $\sim 0.9 \mathrm{~mm}$ increased to $\sim 0.95 \mathrm{~mm}$. The rim was also partially evaporated, which suggests that the rim was also illuminated. The composition of the rim and that of the target material also varied. Confirmed by a Scanning Electron Microscope (SEM) the surface material was initially composted of $(\mathrm{Mg}, \mathrm{Fe})_{2} \mathrm{SiO}_{4}$. However during the ablation event the subsurface inclusions of aluminium and calcium were brought to the surface (i.e. from the bottom of the ablation hole and/or from the material matrix of the sample). These inclusions represent impurities within the target material's inhomogeneous structure [30]. Again confirmed by the SEM the illuminated rim was composed of $(\mathrm{Ca}, \mathrm{Al}) \mathrm{SiO}_{4}$. The sublimation temperature at 1 atmosphere of olivine with calcium deposits is $3800 \mathrm{~K}$ and for $\mathrm{Mg}_{2} \mathrm{SiO}_{4}$ is $3350 \mathrm{~K}$ [31]. This variation will result in the incongruent sublimation of the target material [31]. This will occur over a range of temperature limits and can include the full, partial and un-vaporised sections of the illuminated material [25,31]. The exposure of new material may therefore increase the sublimation temperature of the illuminated material. The laser beam will have to heat the rock to a higher temperature, while also experiencing the effects of a slightly defocused laser beam. Lower pockets of sublimation may also be encountered. This can be caused by the transparency of the pure minerals contained within the target material. The plume of gaseous ejecta will also increase the local pressure surrounding the ablation zone. This will assist in

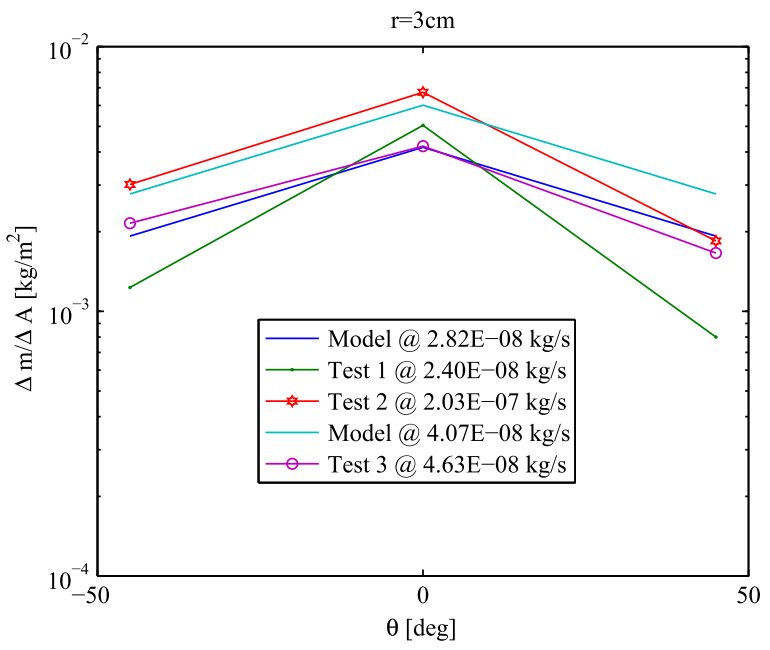

Fig. 5. Mass deposition per unit area: experimental result vs. model prediction at $3 \mathrm{~cm}$ from the spot.
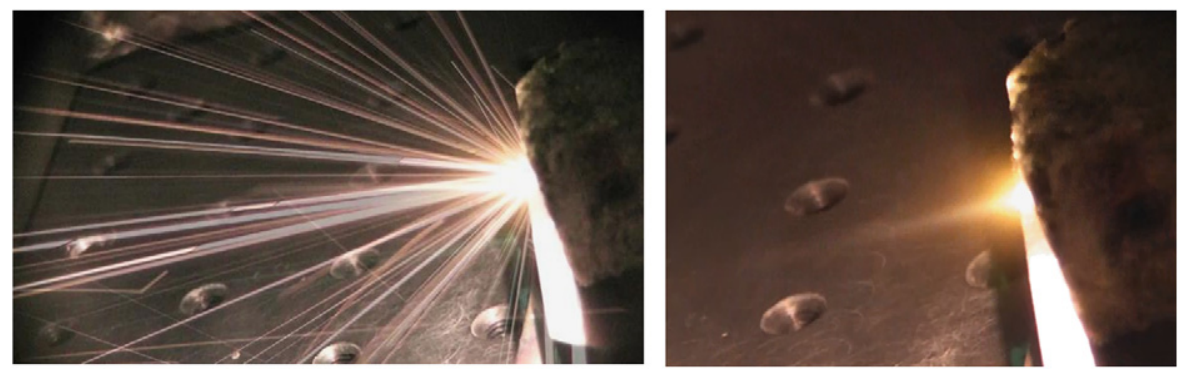

Fig. 4. Ablation response of the olivine sample (Left, with ejecta; Right, mini rocket plume). 


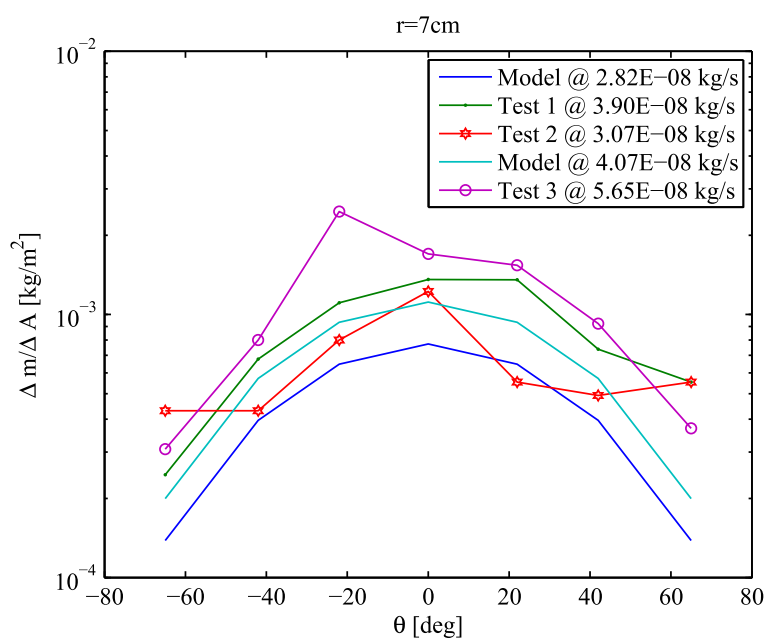

Fig. 6. Mass deposition per Unit Area: Experimental Result vs. Model Prediction at $7 \mathrm{~cm}$ from the Spot.

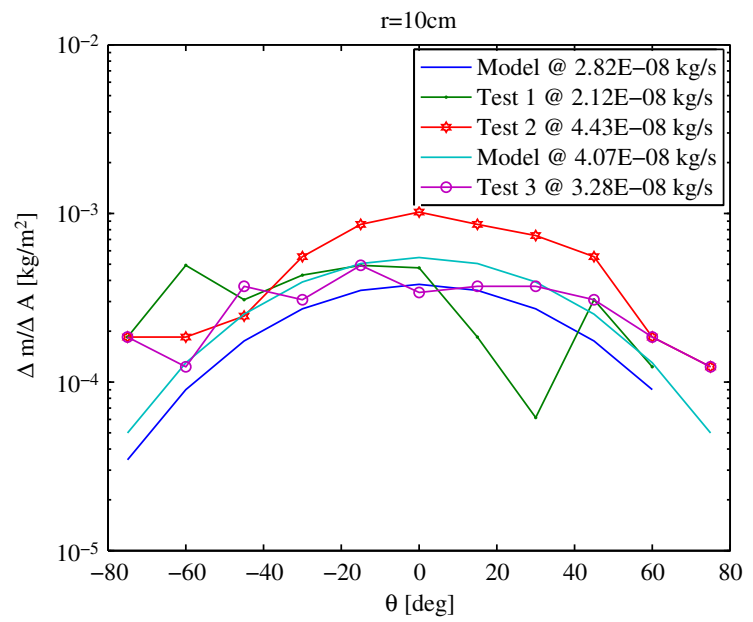

Fig. 7. Mass deposition per unit area: experimental result vs. model prediction at $10 \mathrm{~cm}$ from the Spot.

increasing the temperature of the plume and in partially melting the target material around the ablation rim [32].

The self-cleaning action of the laser beam, as discussed later, would also contribute to the rise in the ablated gas temperature. The laser is considered to either: (1) re-heat and re-sublimate the deposited ejecta [32] and/or (2) reexcite and re-direct any ejecta that enters the laser beam, preventing it from reaching or depositing onto the microscope slides. However each hypothesis needs to be validated further with more experiments and SEM analysis. After the initial ablation event, the ablated particles will also recombine and separate into much simpler molecules and atoms. This will release more energy into the flow and assist in increasing the temperature of the plume [27].

The absorption of the laser radiation into the bulk of the target material also has to be considered. To introduce the actual absorption of the target material the worst case scenario was taken. This was considered to be $1-(0.3 \times 1.2)$. i.e. 0.3 is the worst-case reflectivity of

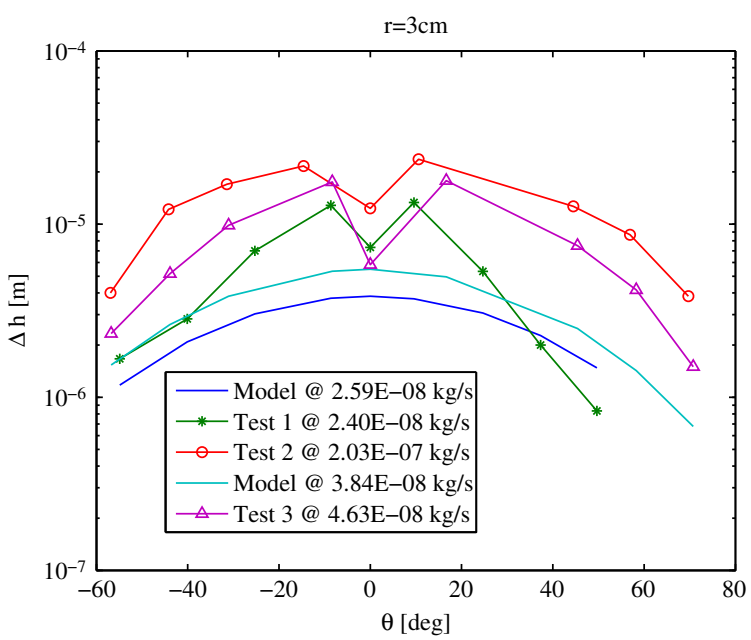

Fig. 8. Thickness of the material deposited on the slides at $3 \mathrm{~cm}$ from the spot. Comparison between experimental measurements and simulation results.

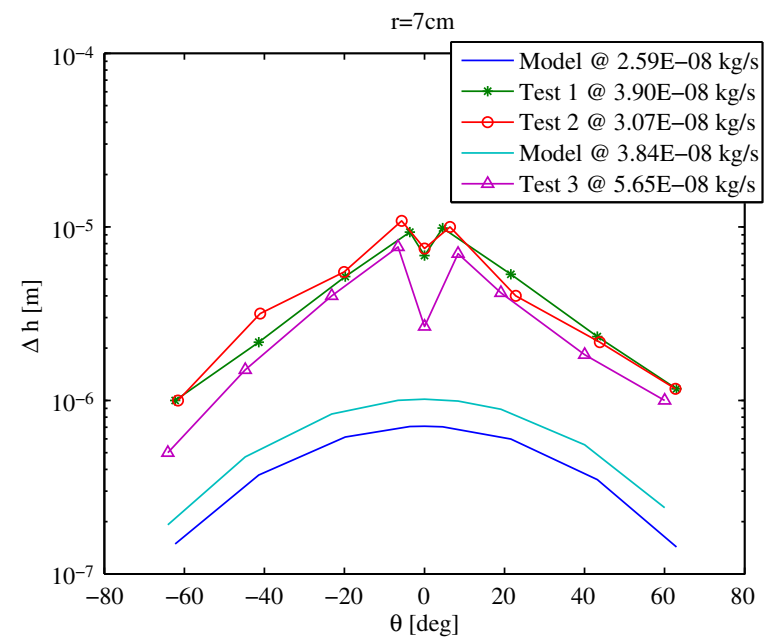

Fig. 9. Thickness of the material deposited on the slides at $7 \mathrm{~cm}$ from the spot. Comparison between experimental measurements and simulation results.

olivine at the critical frequency of the visible range and 1.2 is the peak increment of reflectively at the frequency of the laser $(808 \mathrm{~nm})$. It was also assumed that the gas within the ejecta plume absorbed about $10 \%$ of the incoming laser beam. Therefore the power value as given in Eq. (6) is the power absorbed at the spot. $\eta_{\text {abs }}$ in Eq. (6) accounts for the absorption of the laser beam into the target material and the absorption of gas within the plume of ejecta. It is therefore expressed as $\eta_{\mathrm{abs}}=0.9(1-(0.3 \times 1.2))$.

These cumulative effects would explain the temperature difference between the expected spot temperature $(3100-3800 \mathrm{~K})$ and the result measured by the spectrometer (4285-4747 K). The additional heat absorbed in the Knudsen layer is equivalent to increasing the enthalpy of sublimation by approximately $1-2 * 10^{6} \mathrm{~J} / \mathrm{kg}$ [28] and heating up the gas from 3100 to $4747 \mathrm{~K}$ would consume 


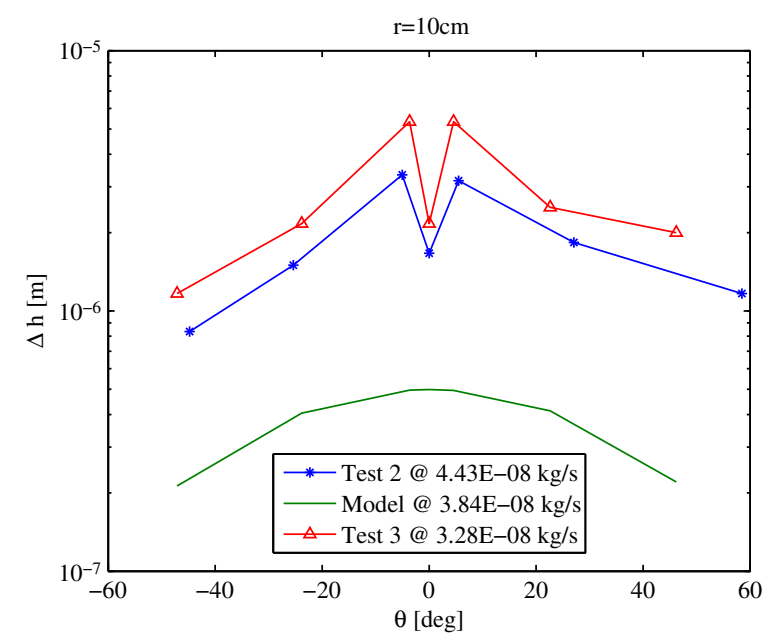

Fig. 10. Thickness of the material deposited on the slides at $10 \mathrm{~cm}$ from the spot. Comparison between experimental measurements and simulation results.

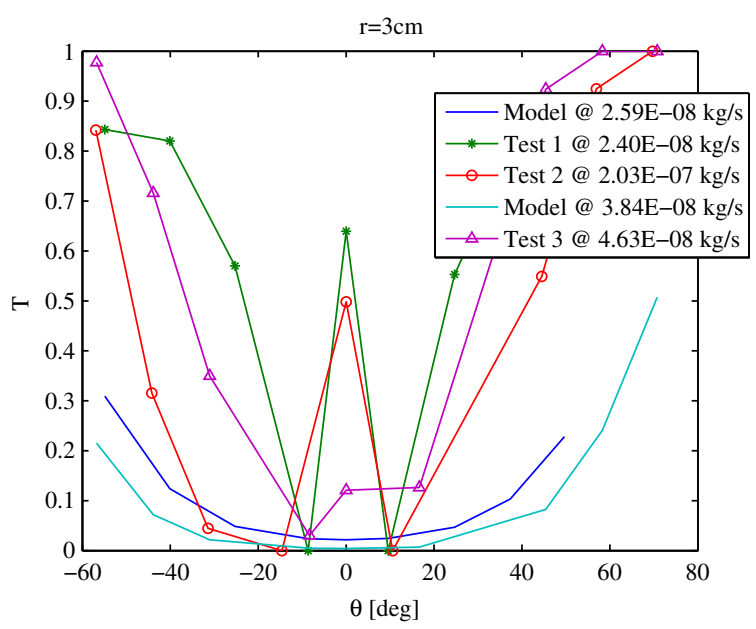

Fig. 11. Degradation factor: comparison between experimental results and model prediction at $3 \mathrm{~cm}$ from the spot.

(assuming a specific heat of $1361 \mathrm{~J} / \mathrm{kg} \mathrm{K}$ ) approximately $2 \mathrm{MW} / \mathrm{m}^{2}$ of energy.

Each experimental test reported in the figures is the average mass flow rate of the ablated target material. The model presented in this paper, predicted a much higher ablated mass flow rate. The inclusion of the formation of the Knudsen layer [30,33,34] and the absorption of energy in the plume provided some corrective factors that allowed for a more accurate prediction of the mass flow rate. However the current numerical model is still limited. It does not account for the three dimensional thermal diffusion effects. This could potentially play an important role in the reduction of the mass flow with time. Further analyses, therefore, needs to be performed to update the simulations with a more accurate model of the energy draining effects.

The efficiency of the ablation process also varied between $0.022 \%$ and $0.148 \%$. This corresponded with a measured momentum coupling coefficient of between

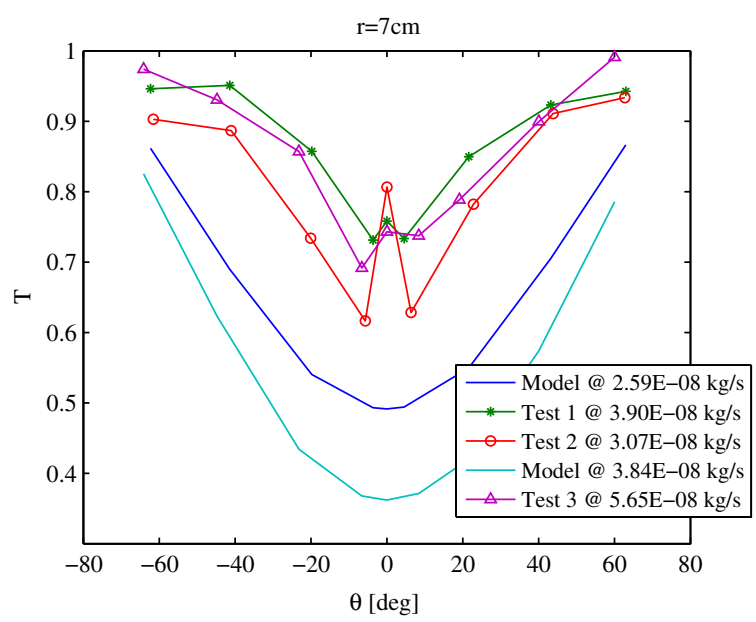

Fig. 12. Degradation Factor: comparison between Experimental results and model prediction at $7 \mathrm{~cm}$ from the spot.

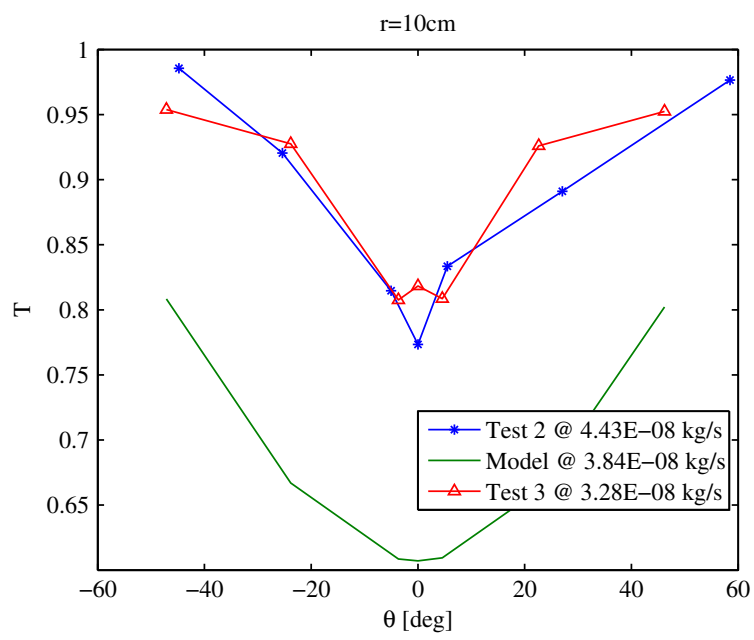

Fig. 13. Degradation factor: comparison between experimental results and model prediction at $10 \mathrm{~cm}$ from the spot.

$6.15 \cdot 10^{-7}$ and $4.10 \cdot 10^{-6} \mathrm{~N} / \mathrm{W}$ and an energy usage of between $1.76 \cdot 10^{8}$ and $1.17 \cdot 10^{9} \mathrm{~J} / \mathrm{kg}$. The range of data is caused by the variation in the mass flow rate of the ablated material. All values given are, in part, calculated from the average ablated mass flow rate (over the ablation period) gained from the experiment. The reported momentum coupling coefficient was considered to be a more important parameter than the energy usage and efficiency of the ablation process. An increase in the momentum coupling coefficient implies an increase in the ablated mass flow rate. Also unlike conventional propulsion-based ablation, the deflection of asteroids through laser ablation is not fuel (i.e. mass) limited. If required the entire asteroid could be ablated. This could provide a potentially endless supply of propellant for the ablation process. However the current show stopper is in the degrading and contaminating effects of the ablated ejecta. This is currently being addressed.

The velocity of the gaseous ejecta plume $\bar{v}$ was calculated to be $\sim 1130.8 \mathrm{~m} / \mathrm{s}$. This was inferred by using the 
temperature of sublimation of the target material (3100$3800 \mathrm{~K}$ ) and assuming Maxwell's distribution of an ideal gas. It should be noticed that the temperature recorded in the experiment was more than the minimum sublimation temperature assumed for the deflection simulations in Section 2.

Figs. 5-7 shows the accumulated mass per unit area for the different experiments (i.e. tests) and at different distances from the spot. The values given correspond to a few discrete, but representative samples that were taken along each microscope slide. Each test produced a different ablated mass flow rate, which is also reported in the figures. It should be noted that the reported mass flow rate as given in each figure is the average value that was experienced over a sublimation period of 10 mins. From inspection it can be observed that there is a direct correlation between the amount of deposited material and the ejected mass flow rate. The input power for test 1 was $43 \mathrm{~W}$ while for test 2 and 3 it was $62 \mathrm{~W}$. For all three distances - either 3,7 or $10 \mathrm{~cm}$ away from the spot - the deposited mass predicted by the model is very similar to the experiment.

Figs. 8-10 shows the thickness of accumulated material. The experimental results show a much higher thickness than predicted by the model, although with a similar variation with the elevation angle. An exception to this is represented by the central point at elevation of zero degrees. This demonstrates that the laser beam has a cleaning effect on the microscope slides. This is partially due to the fact that the particles in the laser beam tend to be excited while travelling towards the microscope slides and partially because they are re-evaporated once deposited. Figs. 11-13 shows the measured degradation of the light intensity going through the microscope slides. Within these figures the experimentally derived degradation factor, as given in $\mathrm{Eq}(10)$ is denoted T. Degradation is caused by the accumulation of the deposited ejecta. This reduces the transmittance

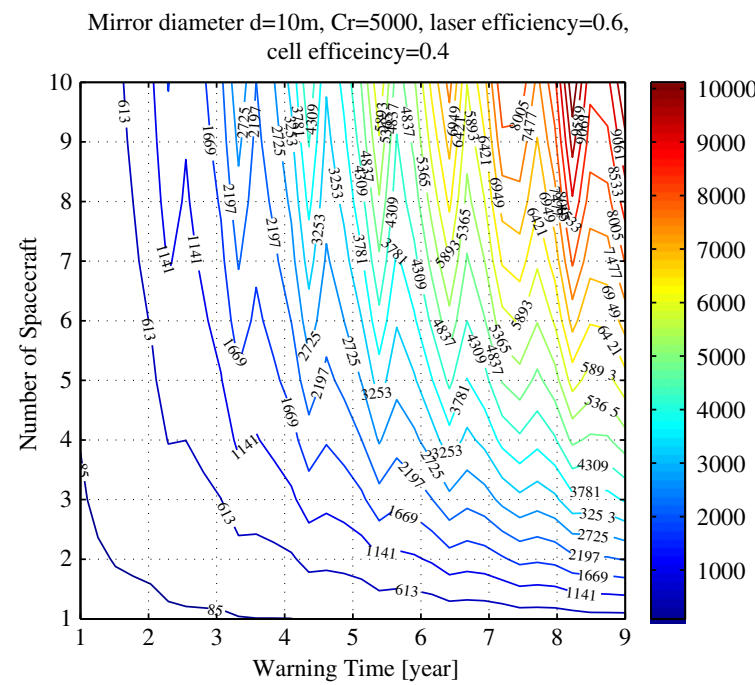

Fig. 14. Revised deflection simulations with the experimentally measured $\eta$. of the exposed surface. The profile of the degradation factor follows closely the distribution of the thickness.

The model predicted the ejecta to result in a significantly greater degradation than was otherwise observed. This degradation is expected to be higher at lower elevation angles, where the plume density is large. The thickness instead is much higher than expected with an equal mass per unit area. The density of the material deposited on the microscope slides is therefore lower than the $1000 \mathrm{~kg} / \mathrm{m}^{3}$ assumed in the simulation model. At 7 and $10 \mathrm{~cm}$ the average density is about $250 \mathrm{~kg} / \mathrm{m}^{3}$. At $3 \mathrm{~cm}$ this is much higher with an average value over the central slide of about $700 \mathrm{~kg} / \mathrm{m}^{3}$. The average absorptivity at 7 and $10 \mathrm{~cm}$ from the spot location is around $5 \cdot 10^{4} \mathrm{~m}^{-1}$. However at $3 \mathrm{~cm}$ from the spot location, on the central slide the average absorptivity increases to about $10^{5} \mathrm{~m}^{-1}$. The absorptivity values then drops off rapidly below $10^{4} \mathrm{~m}^{-1}$ over the two microscope slides positioned at $\pm 45^{\circ}$.

It is therefore reasonable to assume that at $3 \mathrm{~cm}$ from the spot location that the plume is very focussed and that the material is mainly distributed over the central slide. At 7 and $10 \mathrm{~cm}$ from the spot location the plume is more expanded and therefore leads to a more distributed layer of material. In all cases it seems that the model assumes an incorrect growth of the deposited material, an incorrect density and an incorrect absorptivity value. It also has to be noted that the deposited material is not bonded with the slides, i.e. by vibrating the slides most of the accumulated material can be removed.

\subsection{Revised deflection simulations}

Data gained from the experiment campaign demonstrated a good representation to the expected, theoretically predicted mass flow rate. However, the ejecta plume appears to be more focussed than was otherwise predicted by the model. This factor would increase the performance of the deflection method but is dependent on the type of material and the composition of the given asteroid. For a rocky material, composed of olivine it is reasonable to consider the model as being conservative. However, further analysis with different materials is still required to provide full characterisation of the laser ablation process. Note that the temperature of the ablation spot is higher than in the model as the power density is higher. This aspect needs to be investigated further as the model assumes that an increase in power density will increase the mass flow, as it does, but will not increase the temperature of the spot. Theoretically the thrust is only increased by increasing the mass flow rate. It is also interesting that while the laser beam spot size diameter is $1.8 \mathrm{~mm}$, the area of the ablated material is much larger. The diameter of the hole created during ablation increased. This suggests that the ablation process extends beyond the area initially illuminated by the laser.

The experimental analysis has also demonstrated that the contamination due to the re-condensation of the sublimated material is far lower than forecasted in Kahle et al., 2006 [11]. One reason seems to be that the condensed material has a much lower density than 
expected and is different in nature from what was expected. The absorptivity of the condensed ejecta was two orders of magnitude lower than otherwise assumed in the model. Also at $3 \mathrm{~cm}$ away from the spot, it appears that the deposited ejecta were subjected to a much more focussed plume, and that the deposited material appears to be the subject of compression. The situation at $3 \mathrm{~cm}$ is not representative of the conditions in which the spacecraft are supposed to be flying in. The microscope slides positioned at 7 and $10 \mathrm{~cm}$ displays a more representative behaviour. From the light transmittance analysis, the ejecta on the microscope slides positioned at 7 and $10 \mathrm{~cm}$ away from the spot have an average absorptivity value of $5 \cdot 10^{4} \mathrm{~m}^{-1}$ and a density of $250 \mathrm{~kg} / \mathrm{m}^{3}$. Using these values the deflection simulations were re-computed. For direct comparison the initial conditions and the overall efficiencies for the results previously obtained in Figs. 2 and 3 were used. The updated deflection scenario is given in Fig. 14. Using the same mission scenario, but the experimentally determined absorptivity value (rather than assumed) it can be summarised that over a one to nine year operational period the achievable deflection distance varies from 85 to $10,000 \mathrm{~km}$. This is relative to an increase of spacecraft that fly in formation with the asteroid.

Fig. 14 shows a substantial increase in performance. In comparison to Fig. 3, which shows the original and expected level of contamination, the achievable miss distance doubles in magnitude. A maximum deflection distance of $10,000 \mathrm{~km}$ is achieved. This is an increase of performance of $122 \%$. While the effect of the deposited ejecta contamination is still present, it is reduced with respect to the expectations of the model. However it should be noted that although the new simulations, with the updated contamination factor show an increase in performance, it does not take into account the higher sublimation temperature (registered during the experiments) and the additional energy drains. From preliminary calculations, if the additional energy drains and a sublimation temperature (in the $3100-3800 \mathrm{~K}$ range) are considered then, to achieve an equivalent performance the concentration ratio would need to be increased to 60000. Also note that, during each experiment the microscope collection plates were at room temperature $\left(25^{\circ} \mathrm{C}\right)$. It is therefore reasonable to expect that a higher temperature might decrease the condensation of material. One possible method to reduce the effect of contamination of the plume would be to maintain the collectors at a moderately high temperature, over $100{ }^{\circ} \mathrm{C}$.

\section{Conclusion}

The experiments performed within this paper enabled the current modelling technique for laser ablation to be assessed. While the experimentally measured and theoretically predicted mass flow rates of the target material and deposition rate of the ejecta compared well to each other, the nature of the deposited ejecta was significantly different. The experimentally measured absorptivity values were two orders of magnitude lower than assumed in the model. The density of the deposited ejecta was also reduced. The model therefore assumed an incorrect growth of the deposited material, density and absorptivity of the condensed material. It also assumed that all the ejected material would bind with any exposed surface. This was determined not to be the case. These parameters therefore represent a current inaccuracy within the existing modelling technique. This will otherwise affect the endurance, efficiency and overall response of any ablation based asteroid deflection and mitigation mission. Ejecta contamination will always affect any laser ablation based mission. However the experiment also demonstrated that the effect of the contaminating deposited ejecta was far less than predicted in the model. This results in a comparatively larger level of achievable deflection, where there is no immediate saturation of any exposed surface. However, further work is still required to assess the laser ablation process. A wider range of asteroid analogue target material intends to be tested. This includes a highly porous sample and a collection of meteorites. The inhomogeneous nature of the target material, and that of the asteroid, must also be accounted for. Different compounds will ablate at a lower and higher sublimation temperature. The model also needs to be developed to account for the three dimensional thermal diffusivity and the temperature dependence on the optical and thermal properties of the target material. This includes the emissivity, heat capacity, density and thermal conductivity. The non-thermal emissions created by the ablated gas particles also needs to be considered. More experiments are therefore required to further explain and develop the mathematical model and existing discrepancies.

Once proven successful for a range of compositions present within the asteroid, and small body population, the role of laser ablation could be extended for a number of space-based applications. The laser ablation process itself effectively tunnels into the illuminated material, extracting material in the form of an ejecta plume. This results in the extraction of deep and previously inaccessible material that could not otherwise be retrieved through conventional in-situ and sample return based missions. The material extracted from laser ablation could be collected externally by the spacecraft flying through the plume. The collected ablated material could then be used as part of a sample return mission and/or for resource exploration and exploitation. In-situ spectra analysis of the ablated ejecta plume could also be performed. Laser ablation therefore provides a novel technique for the interaction and collection of material from a small, rocky body. It is also considered to be advantageous as it provides a contact-less and remote method of analysis that eliminates any mission requirement to physically land and/or attach itself to the asteroid. For asteroid deflection purposes it also avoids any possible fragmentation of the asteroid. Laser ablation could enable scientists and engineers to further characterise the composition, formation and evolution of asteroids and other small bodies.

\section{Acknowledgements}

This work has been sponsored by The Planetary Society, USA. Work has been conducted by the Advanced Space Concepts Laboratory at the University of 
Strathclyde, the Institute of Photonics, University of Strathclyde and the School of Engineering, University of Glasgow. Thank you to Mr. Noble for reviewing this document and to the support given by Mr. Hugh Parker.

\section{References}

[1] J.W. Campbell, C. Phipps, L. Smallley, J. Reilly, D. Boccio, The Impact Imperative: Laser Ablation for Deflecting Asteroids, Meteoroids, and Comets from Impacting the Earth APS Conference Proceeding 664 -First International Symposium on Beamed Energy Propulsion, American Institute of Physics, Melville, NY, 2003, pp. 509-520.

[2] H.J. Melosh, I.V. Nemchinov, Y.I. Zetzer, Non-nuclear strategies for deflecting comets and asteroids, in: T. Gehrels (Ed.), Hazards Due to Comets and Asteroids, University of Arizona Press, Tucson, 1994 pp. 1111-1132.

[3] B.A. Conway, Near-optimal deflection of earth-approaching asteroids, J. Guid. Control Dyn. 24 (5) (2001) 1035-1037.

[4] C. Gritzner, R. Kahle, Mitigation technologies and their requirements, in: M. Belton, T. Morgan, N. Samarainha, D. Yeomans (Eds.) Mitigation of Hazardous Comets and Asteroid, Cambridge University Press, Cambridge, 2004, pp. 167-200.

[5] J.P. Sanchez, C. Colombo, M. Vasile, et al., Multicriteria comparison among several mitigation strategies for dangerous near-earth objects, J. Guid. Control Dyn. 32 (2009) 121-141.

[6] M. Vasile, C. Maddock, On the deflection of asteroids with mirrors, Celestial Mech. Dyn. Astron. 107 (2010) 265-284

[7] M., Vasile, C., Maddock, G., Radice, McInnes C., NEO Deflection though a Multi-Mirror System, ESA Call for Proposals: Encounter 2029, Final Report for Ariadna Study Contract 08/4301, Technical officer: L. Summerer, 2009.

[8] M. Belton, T.H. Morgan, N. Samarasinha, D.K. Yeomans, Mitigation of Hazards Comets and Asteroids, Cambridge University Press, United Kingdom, 2004

[9] C.A. Maddock, M. Vasile, C. Mcinnes, et al., Designs of Multispacecraft Swarms for the Deflection of Apophsis by Solar Sublimation, Planetary Defence Conference, Spain, 2009.

[10] C., Maddock, M. Vasile, L, Summerer, Conceptual Design of a Multi-Mirror System for Asteroid Deflection, Paper Presented a the 27th International Symposium on Space Technology and Science, Epochal Tsukuba, Japan, 2009.

[11] R. Kahle, E. Kuhrt, G. Hahn, J. Knolenberg, Physical limits of solar collectors in deflecting earth-threatening asteroids, Adv. Sci. Technol. 10 (2006) 256-263.

[12] N.I. Komle, Jet and shell structures in the cometary coma: modelling and observations, in: J.W. Mason (Ed.), Comet Halley: Investigation, Results, Interpretations, vol. 1, 1990, pp. 231-244.

[13] C. Phipps, M. Birkan, W. Bohn, et al., Review: laser ablation propulsion, J. Propul. Power 26 (4) (2010) 609-637.

[14] C. Phipps, J. Luke, Diode laser-drive microthrusters: a new departure for micropropulsion, AIAA 40 (2) (2002) 310-318.

[15] J.E.M. Sinko, C. Phipps, Modelling $\mathrm{Co}_{2}$ laser ablation impulse of polymers in vapour and plasma regime, Appl. Phys. Lett. 95 (2009) 121105.

[16] P. Bhargave, M. Kumar, H. Kumar, et al., Impulse coupling in laserdriven microtargets, J. Phys. 62 (4) (2003) 923-932.

[17] G. Dettleff, Plume flow and plume impingement in space technology, Aerosp. Sci. 28 (1991) 1-71

[18] C. Colombo, M. Vasile, G. Radice, Semi-analytical solution for the optimal low-thrust deflection of near-earth objects, J. Guidance, Control Dyn. 32 (2009) 796-809.

[19] C.R. Phipps, Laser deflection of near-Earth asteroids and comet nuclei, in: Proceedings of the International Conference on Lasers 96 STS Press, McLean, VA, 1997, pp. 580-587.

[20] S.M. Yoo, Y.M. Song, S.Y. Park, Spacecraft formation flying for earthcrossing object deflections using a power limited laser ablating, Adv. Space Res. 43 (12) (2009) 1873-1889.

[21] W.O. Shall, H.A. Eckel, W.L. Bohn, Laser propulsion thrusters for space transportation, in laser ablation and its applications, Springer Series of Optical Science 129 (2007) 435-454.

[22] A. Gibbings, J.M. Hopkins, D. Burns, M. Vasile, I. Watson, On testing laser ablation processes for asteroid deflection, in; Proceedings of IAA Planetary deference conference, 9-12 $2^{\text {th }}$ May, Bucharest, Romania, 2011.

[23] L.W. Alvarez, W. Alvarez, F. Asaro, H. Michel, Extra-terrestrial causes for the cretaceous-tertiary extinction, Science 208 (1980) $1095-1108$.
[24] H. Legge, R.D. Boettcher, Modelling control thruster plume flow and impingement, in: Proceeding of the 13th International Symposium on Rarefied Gas Dynamics, Novosibirsk, (1982), pp. 983-992.

[25] J. Lindgrad, K. Jonansen, Production and testing of concrete with high specific heat, P. Bartos, D.L. Marrs, D.J. Cleland (Eds.) in: Proceedings of the International RILEM Conference, Paisley, Scotland, June 3-5, 1996.

[26] M.M. Marinova, O. Aharonson, E. Asphaug, Geophysical consequences of planetary-scale impacts into a Mars-like planet, Icarus 211 (2011) 960-985.

[27] B.R. Pabani, K.A. Pathak, CFD analysis of laser ablation for nanotube production - a review, Int. J. Eng. Res. Appl. 2 (2012) 1204-1207.

[28] C.J. Knight, Theoretical modeling of rapid surface vaporization with back pressure, AIAA 17 (1979) 519-523.

[29] S.I. Anisimov, B.S. Luk'yanchuk, Selected problems of laser ablation theory Physics 43 (3) (2002) 293-324.

[30] P.S.P. Wei, D.J. Nelson, R.B. Hall, Laser-induced evaporation of solid surfaces, J. Chem. Phys. 62 (8) (1975) 3050-3051.

[31] T.J. Ahrens, J.D. O'Keefe Shock, Melting and vaporization of lunar rocks and minerals, Earth Moon Planets 1 (1) (1972) 214-249.

[32] C.D. Boley, J.T. Early, Computational model of drilling with high radiance pulsed lasers, Presented at the applications of lasers and electro-optics, Orlando, FL, 17-20 Oct.(1994).

[33] C.J. Knight Theoretical modelling of rapid surface vaporization with back-pressure in: Proceedings of the 11th AIAA Fluid and plasma dynamics conference, Seattle, USA, July 10-12, (1978).

[34] H. Fujisawa, N. Fujii, H. Mizutani, H. Kanamori, S. Akimoto, Thermal, Diffusivity of $\mathrm{Mg}_{2} \mathrm{SiO}_{4}, \mathrm{Fe}_{2} \mathrm{SiO}_{4}$, and $\mathrm{NaCl}$ at high pressures and temperatures, J. Geophys. Res. 73 (14) (1986) 4727-4733.

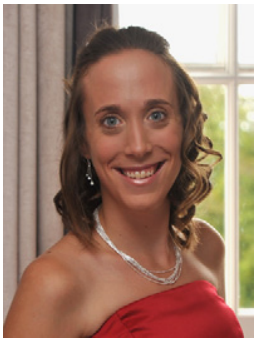

Alison Gibbings is currently a Ph.D. candidate within the Advanced Space Concepts Laboratory (ASCL) at the University of Strathclyde. Her Ph.D. research is focussed on the interception and deflection of Near Earth Asteroids. This is from both an experimental and analytical approach. Prior to joining the ASCL, Alison completed a Young Graduate Traineeship at ESA/ESTEC. This involved working on developing planetary missions in preparation for the future human exploration of the Moon, and other planetary bodies. Alison has an M.Eng. (Hons.) in Aerospace Engineering and Astronautics from Kingston University, London.

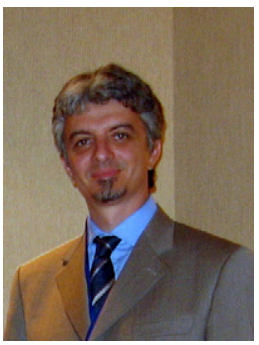

Massimiliano Vasile is the Associate Director of the Advanced Space Concepts Laboratory, and Reader in the Mechanical \& Aerospace Engineering Department, both at the University of Strathclyde. Previous to this he was a Senior Lecturer in the Department of Aerospace Engineering and Head of Research for the Space Advanced Research Team at the University of Glasgow. His main research interests are Space Mission Analysis and Design, Computational Optimization, Robust Design Optimization and Uncertainty Quantification, Multidisciplinary Design, Asteroids, Space Flight Mechanics, and Autonomous Robotic Systems. $\mathrm{He}$ is member of the IAF Space Power Committee.

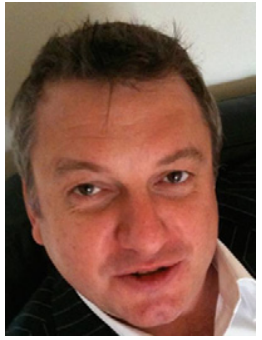

lan Watson's first degree was in Applied Physics, followed by a Ph.D. from the University of Glasgow in "Optimising the Gaseous Discharge and Optical Coupling of a Pulsed $\mathrm{CO}_{2}$ Laser", specifically designed for material processing reflective and refractory materials. In the 1990s he began to research the effects of high power lasers on microorganisms, laser sterilisation and inactivation; publishing on their direct effects and efficacy on treating different substrates, including solids, liquids and air and a range of microorganisms from E. coli to B. globigii, an anthrax simulant. He is also developing algae growth and oil extraction systems for biofuel production. 


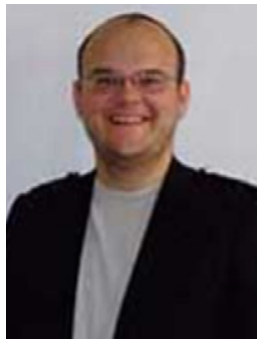

John-Mark Hopkins is an Associate Team Leader at the Institute of Photonics (IoP). His research interests are in developing highpower semiconductor laser sources in the near infrared, and in the development of novel and compact all-solid-state femtosecond laser sources. Before joining the IoP John-Mark was a Postdoctoral Research Fellow at the University of St Andrews within the Semiconductor Physics Group. His research was on pump-probe investigations of semiconductor optical amplifiers (SOAs) at near and mid-infrared wavelengths. He received both his Ph.D. and M.Sc. in Laser Physics from the University of St Andrews, Scotland.
David Burns is an Associate Director at the Institute of Photonics (IoP). He was awarded his Ph.D. from the University of St. Andrews in 1990 for work with Professor Wilson Sibbett on semiconductor and fibre lasers. From 1990 to 1996, he was a research fellow in Prof. Sibbett's group working on optical pump sources and ultrashort pulse generation. Joining the Institute of Photonics, University of Strathclyde in 1996, he has led the Solid-State Laser Development group since 2002. His research interests include: optically-pumped semiconductor disk, highpower SBR modelocked solid-state and mid-infrared lasers; stabilisation techniques, adaptive optics, and; the development of practical laser systems. 